\title{
Mechanical ventilation affects the microecology of the rat respiratory tract
}

51 Department of Anesthesiology, Nanfang Hospital, Southern Medical University, Guangzhou, $6 \quad$ Guangdong, China.

7 2. Guangdong Provincial People's Hospital, Guangdong Academy of Medical Sciences , Guangzhou, $8 \quad$ Guangdong, China.

9 Corresponding author: Xiao Jin-fang, Department of Anaesthesiology, Nanfang Hospital, Southern 10 Medical University, Jingxi Street, Guangzhou 510515, Guangdong, China. Email: 11 jinfangxiao100@163.com. 
Abstract

Background The most common 'second strike' in mechanically ventilated patients is a pulmonary

infection caused by the ease with which bacteria can invade and colonize the lungs due to mechanical

ventilation. At the same time, metastasis of lower airway microbiota may have significant implications

in the development of intubation mechanical ventilation lung inflammation. Thus, we establish a rat

model of tracheal intubation with mechanical ventilation and explore the effects of mechanical

ventilation on lung injury and microbiological changes in rats.

Methods Sprague-Dawley rats were randomized into control, Spontaneously Breathing (1, 3, 6 hours),

21 Mechanical ventilation(1, 3, 6 hours) groups. Lung wet to dry weight ratio (W/D weight ratio) and Lung

histopathological injury score were evaluated.16SrDNA sequencing was performed to explore

respiratory flora changes.

Results Bacterial diversity was comparable between healthy and intubation mechanical ventilation

rats, with time relation. Ordination analyses revealed that samples clustered more dispersing by

tracheal intubation and mechanical ventilation. Finally, predicted metagenomes suggested a

substantial increase in biofilm formation phenotype during early tracheal intubation and mechanical

ventilation.

Conclusion Collectively, these results establish a link between the duration of mechanical ventilation

and alterations to the respiratory tract microecology. In future studies, we hope to discover the 
bioRxiv preprint doi: https://doi.org/10.1101/2021.12.07.471701; this version posted December 9, 2021. The copyright holder for this preprint (which was not certified by peer review) is the author/funder. All rights reserved. No reuse allowed without permission.

34 Keywords: Mechanical ventilation, lung injury, Respiratory tract, Microecology, Floraimbalance 


\section{Introduction}

Ventilator mechanical ventilation is widely used to manage general anesthesia during surgery, respiratory maintenance in intensive care, and perioperative treatment of critically ill patients. However, mechanical ventilation can also lead to lung injury or exacerbate existing lung injury, known as ventilator-induced lung injury (VILI) ${ }^{1,2}$. Also, lung inflammation can occur during ventilator therapy. Current studies have found that mechanical ventilation induces upregulation of cytokine expression in

a pro-inflammatory state to the body. Thus, patients are more susceptible to "second strikes" 44 (prolonged mechanical ventilation, aspiration, shock, sepsis, pulmonary infections) ${ }^{3}$. The most common "second strike" in mechanically ventilated patients is pulmonary infections. The effects of the body's immune system from the own comorbidities and malnutrition in patients receiving mechanical ventilation under anesthesia6 can also increase the morbidity and mortality of respiratory infections ${ }^{4}$. Respiratory microecology is one of the critical factors in the function of the respiratory tract. Studies over the past few years have demonstrated that the lower respiratory tract is not "sterile" and that, in healthy conditions, the lung microbiota is less dense but harbors a remarkable diversity of interacting microbiota. The ribosomal DNA of Actinobacteria, Aspergillus, Bacteroides, and Bacteroides is present 52 in the lungs of healthy individuals ${ }^{5-8}$. The "steady state" of the lung microbiome during health may be a process of continuous influx and continuous elimination of unfavorable growth conditions. Studies have confirmed that the balance between immigration and elimination during pulmonary disease is

55 disturbed. The pulmonary microbiota is altered, with bacteria exhibiting competitive dominance ${ }^{9}$, 56 resulting in an imbalance in the host immune system ${ }^{7,10-12}$. 
57 The imbalance of respiratory flora may lead to local or even systemic bacterial infections, and the

58 microecological regulatory mechanisms are complex. Identification of microbial colonization by

59 alveolar lavage collected primarily from critically ill patients is currently used clinically to guide

60 anti-infection protocols, while few studies have been reported on the relationship between lung injury

61 and inflammatory response to mechanical ventilation and changes in respiratory flora. Therefore, this

62 study investigated the relationship between lung injury and microbiological changes in rats with

63 tracheal intubation mechanical ventilation and contributed to the study of flora regulation of the 64 respiratory tract in lung injury and inflammation. 


\section{Materials and Methods}

To investigate the relationship between lung injury from mechanical ventilation and changes in respiratory flora, we assessed the inflammatory response and microbial changes in the rat airways by pathophysiology and 16SrDNA sequencing.

\section{$71 \quad 2.2$ Animals}

Male Sprague - Dawley rats (weighing between 230-330g and aged 8-9 wk) were housed at the

Southern Hospital Experimental Centre of Southern Medical University, under the same temperature and humidity environment, and received the same food and water. Animal care and experimental protocols were guidelines by the National Science Council of the Republic of China(NSC1997). The animal studies were reviewed and approved by the Ethical Committee on Animal Experimentation of Nanfang Hospital, Southern Medical University, Guangzhou, China ( NFYY-2021-0245 ) . Twenty-eight male SD rats were randomly divided into seven groups $(n=4)$ : control group (group $C)$, tracheal intubation with spontaneous breathing for one hour (group SV1), tracheal intubation with spontaneous breathing for three hours (group SV3), tracheal intubation with spontaneous breathing for six hours (group SV6), tracheal intubation with mechanical ventilation for one hour (group MV1), tracheal intubation with mechanical ventilation for three hours (group MV3), tracheal intubation with mechanical ventilation for six hours (group MV6) (MV6 group). After weighing, we anesthetized the rats with $2 \%$ pentobarbital sodium $(60 \mathrm{mg} / \mathrm{kg}$ ) by intraperitoneal injection. We placed our homemade tracheal into the trachea and connected a small animal ventilator (SuperV1.0, HYB, China) for 
mechanical ventilation. A room temperature of $26-28^{\circ} \mathrm{C}$ was maintained throughout the procedure. The

control group was directly executed after tracheal intubation, while the SV group was intubated and left

to breathe independently. The MV group was mechanically ventilated at 1,3 , and 6 hours, respectively.

Mechanical ventilation parameters: tidal volume (VT) $7 \mathrm{ml} / \mathrm{kg}, \mathrm{PEEP}=0 \mathrm{mmHg}$, respiratory rate 80

breaths/min (adjusted according to respiration during anaesthesia), inhalation-expiration ratio (I: E) =

$91 \quad 1: 2$, inhaled gas was air (oxygen concentration $21 \%$ ).

\subsection{Experiments}

\subsubsection{Rat lung injury assay}

\subsubsection{Lung tissue W/D}

95 To investigate the effect of the duration of mechanical ventilation on the edema of lung tissue, we 96 calculated the wet to dry weight ratio (W/D) value of lung tissue. The tissue was weighed on an 97 electronic balance and recorded as wet weight. The tissue was then baked in an oven at $80^{\circ} \mathrm{C}$ and 98 weighed to a constant weight, and recorded as dry weight, and the lung tissue was baked for $\geq 48 \mathrm{~h}$.

\subsubsection{Histopathological examination of the lung}

To observe the pathological damage of lung tissue at different ventilation duration, we performed HE-stained light microscopy of lung tissue. The anterior lobe of the right lung of rats was fixed in $4 \%$ paraformaldehyde solution. After paraffin embedding, sectioning, and HE staining, the lung was placed under a light microscope to observe the histopathological changes. Five high magnification views were randomly selected to observe the lung tissue morphology, and the pictures were taken and saved. 
Scores included lung tissue congestion, septal edema, erythrocyte infiltration, alveolar cavity

destruction, capillary destruction, and other pathological changes, and the mean values were taken after scoring ${ }^{13,14}$.

\subsubsection{Detection of respiratory flora in rats}

\section{Specimen collection}

The trachea and left lung of 28 SD rats were removed, snap-frozen in liquid nitrogen, and stored in a $-80^{\circ} \mathrm{C}$ refrigerator to sequence the $16 \mathrm{SrDNA}$ colonies.

\section{PCR amplification}

The 16S rDNA target region of the ribosomal RNA gene were amplified by PCR ( $95{ }^{\circ} \mathrm{C}$ for 5 min, followed by 30 cycles at $95^{\circ} \mathrm{C}$ for $1 \mathrm{~min}, 60^{\circ} \mathrm{C}$ for $1 \mathrm{~min}$, and $72^{\circ} \mathrm{C}$ for $1 \mathrm{~min}$ and a final extension at $72{ }^{\circ} \mathrm{C}$ for $7 \mathrm{~min}$ ) using primers listed in the table ${ }^{15} . \mathrm{PCR}$ reactions were performed in triplicate $50 \mu \mathrm{L}$ mixture containing $10 \mu \mathrm{L}$ of $5 \times \mathrm{Q} 5 @$ Reaction Buffer, $10 \mu \mathrm{L}$ of $5 \times \mathrm{Q} 5 @$ High GC Enhancer, $1.5 \mu \mathrm{L}$ of 2.5 mM dNTPs, $1.5 \mu \mathrm{L}$ of each primer $(10 \mu \mathrm{M}), 0.2 \mu \mathrm{L}$ of Q5@ High-Fidelity DNA Polymerase, and 50 ng of template DNA. Related PCR reagents were from New England Biolabs, USA.

\section{DNA sequencing and analysis}

Illumina Novaseq 6000 sequencing Amplicons were extracted from 2\% agarose gels and purified using the AxyPrep DNA Gel Extraction Kit (Axygen Biosciences, Union City, CA, U.S.) according to the manufacturer's instructions and quantified using $A B I$ StepOnePlus Real-Time PCR System (Life

Technologies, Foster City, USA). Purified amplicons were pooled in equimolar and paired-end sequenced (PE250) on an Illumina platform according to the standard protocols. The raw reads were deposited into the NCBI Sequence Read Archive (SRA) database (Accession Number: SRP ${ }^{\star * \star \star \star *}$ ). 


\subsubsection{Statistical Methods}

127 For the ratio of Lung tissue W/D and lung injury score, One-way ANOVA test was completed. For

128 Observe species index and Simpson index, comparisons were performed by the Turkey HSD test. The 129 significant difference of Beta diversity was tested with Adonis non-parametric test. The Turkey HSD 130 test compared the relative abundance of respiratory microbiota between groups. P-values less than 0.05 were considered statistically significant. One-way ANOVA test was performed by SPSS software, version 25.0 (IBM Corp, New York, NY, USA). The Adonis non-parametric test was performed, and the Turkey HSD test was performed by the OmicShare tools, a free online platform for data analysis 134 (http://www.omicshare.com/tools). 


\section{Results}

Comparison of lung tissue W/D values between groups

The W/D values of lung tissue in the SV and MV groups were significantly higher after three hours. $(\mathrm{P}<$ 0.05) (Table 1).

\section{Pathological changes in lung tissue}

The alveolar structure of group C was normal, with a small number of inflammatory cells infiltrating the lung. Six hours into the SV group, there was significant hemorrhage, widening of the alveolar septum, lymphocytic infiltration, and massive destruction of the alveolar structure. Three hours into the MV group, diffuse intra-pulmonary hemorrhage was seen. Six hours into ventilation, massive diffuse hemorrhage was seen, widening of the alveolar septum, more exudate in the alveolar cavity, destruction of the tissue structure, fusion of the alveoli and solid lung tissue. The degree of lung tissue damage in the SV and MV groups increased with time (Figure 1).

\section{Analysis of respiratory microbiological changes}

\section{Analysis of the composition of the respiratory flora}

Dominant species largely determine the ecological and functional structure of the microbial community and understand the community's species composition. We analyzed the bacterial composition using $16 S$ rDNA gene sequencing. After filtering and screening at uniform depth, a total of 24 phyla and 244 
genera were identified in the entire sample. To investigate the phylum and genus that accounted for most sequences, we selected species ranked top10 in abundance mean among all samples to show in detail. Others refer to other known species in the figure, and unclassified refer to unknown species ${ }^{16,17}$. The dominant phyla of rats were Proteobacteria, Firmicutes, Bacteroidetes, Cyanobacteria, Actinobacteria. In healthy rats, the dominant genus was Lactobacillus. In mechanically ventilated rats, the doaminant genus was Acinetobacter at the beginning. The proportion of the dominant genus Acinetobacter decreased, and that of Prevotella_9 increased as the duration of ventilation increased. The predominant genus in the respiratory tract of the rats was also Acinetobacter at the early stage of tracheal intubation, while the proportion of Streptococcus gradually increased with time (Figure 2a. Figure 2b). To further investigate the main flora specific to each group, we applied LEFse to analyze the differences in flora between the seven rat groups ${ }^{18}$. As shown in the figure(Figure 2c), only the normal rats and the Ghours group showed the main differential flora, Parvibacter for 6 hours of autonomic breathing, Megamonas for 6hours of mechanical ventilation, and normal rats Acinetobacter_radioresistens.

As expected, the two operations of tracheal intubation and mechanical ventilation resulted in alterations in the respiratory microecology of rats. The main strains of normal adult BALF are Actinobacteria, Proteobacteria, Bacteroidetes, and Firmicutes ${ }^{5-8}$. The strains of rats in this study cover the main human strains and are similar to humans. It has been demonstrated that representatives of the genus Acinetobacter come from the lung microbiome of mechanically ventilated patients with suspected pneumonia ${ }^{19}$. In the present study, the elevated proportion of Acinetobacter as the dominant species during the initial phase of mechanical ventilation with tracheal intubation may 
have contributed to the gradual pathological damage of lung tissue. As the duration of ventilation increased, the proportion of Prevotella_9 gradually increased, and it was considered that the subclinical lung inflammatory manifestations caused by mechanical ventilation created an environment for Prevotella_9 to be retained in the lungs ${ }^{20,21}$. Two classes of probiotics, Parvibacter and Megamonas, are often present in the intestinal tract of humans and animals, and the induction of mechanical ventilation by tracheal intubation itself may be associated with changes in intestinal microbial composition and diversity. There is now strong evidence that the gut microbiota can influence the microbiology of the lung through direct inoculation of bacteria or the distribution of SCFAs ${ }^{22,23}$. Characterization of lower airway probiotic bacteria associated with lung injury from tracheal intubation with mechanical ventilation may be necessary for in-depth exploration of methods to prevent such injury.

Interestingly, although many taxa are shared between samples, the absence of pathogens (e.g., dominant genera) in one sample does not predict their absence in paired sample sequence data. For example, samples V1-1 and V3-1, despite not detecting Sediminibacterium and Curvibacter, showed trends consistent with other genus compared to controls. It is important to note that the dominant genus in samples V1-2 was Streptococcus and the second-highest proportion was Acinetobacter. These examples show that different pathogens can vary in different individuals of the same organism, and therefore, 16S rDNA sequence data are not always representative of lower respiratory tract infections. Lower airway Bacterial diversity varies in healthy and ventilator-assisted breathing rats.

\section{Alpha Diversity}

As mentioned above, in each sample, a few taxa dominate the majority of the sequences. Two alpha 
diversity metrics, Observed species, and Simpson diversity were used to investigate this issue further

to measure species richness and homogeneity ${ }^{24,25}$. The OTU was highest in the mechanically

ventilated 6hours group, indicating the highest abundance. However, the difference between the

control and autonomic breathing 6hours groups was not significant, and OUT abundance decreased at 1 hour and 3hours in both ventilated and autonomic breathing groups (Figure 3a). Using the Simpson diversity index, the abundance and homogeneity of the respiratory flora decreased in each experimental group compared with the control group, but the differences were not significant (Figure $3 b$ and Figure $3 c)$. These data suggest that mechanical ventilation tracheal intubation alters the abundance and homogeneity of lower airway microecology with time influenced.

\section{Beta Diversity}

Beta diversity of the respiratory microbiota was visualized using a ranking method. We were interested in whether the bacterial community aggregates tightly due to tracheal intubation or mechanical ventilation. Previous evidence suggests that three main factors determine the composition of the lower respiratory microbiota, namely microbial migration, microbial elimination, and the relative replication rate of its members. ${ }^{9}$. The oral and pulmonary have similar microbiomes community ${ }^{1,9,26}$. A study has investigated that the oral microbiome is part of the homeostatic processes regulating lung inflammation ${ }^{26}$. Mechanical ventilation operations with tracheal intubation may dysregulate microecology by impairing lower airway defense mechanisms ${ }^{27,28}$. We hypothesized that samples are more intensively clustered by tracheal intubation and mechanical ventilation based on previous studies. To confirm this hypothesis, we used unweighted Unifrac and weighted Unifrac, two abundance-sensitive, phylogenetically relevant diversity metrics, to compare samples ${ }^{29}$. The two 
metrics were calculated to determine the pairwise phylogenetic distances between each sample, then plotted using principal coordinate analysis (PCoA) ${ }^{30}$. From the unweighted Unifrac, contrary to our hypothesis, samples from the SV and MV groups did not cluster more closely with healthy rats (PERMANOVA, $P=0.001$ ) (Figure 4a). The graph shows that the mechanically ventilated group (PERMANOVA, R2=0.3737, $\mathrm{P}=0.002$ ) and the autonomously ventilated group (PERMANOVA $\mathrm{R} 2=0.4702, \mathrm{P}=0.002$ ) both exhibited moderate phylogenetic differences(Figure 4b,Figure 4c). Although experimental groups were better aggregated with healthy rats in terms of weighted Unifrac (PERMANOVA, $P=0.122$ ), the autonomic breathing group exhibited more significant phylogenetic differences than the mechanically ventilated group (PERMANOVA, R2=0.6939, $P=0.001$ ) (Figure 4d, Figure 4e, Figure 4f). These analyses suggest that the overall community structure of the rat lower airway is influenced by tracheal intubation and mechanical ventilation.

\section{Predicted functional categories（功能预测分析 )}

To gain insight into the function of the bacterial community, PICRUSt2 $2^{31}$ was implemented to infer bacterial metagenomes based on 16S rDNA gene content. There were 33 unique KEGG pathways among the inferred metagenomes and showed more remarkable similarities between normal rats and each ventilated group (Figure 5). This shows that mechanical ventilation by tracheal intubation does not significantly alter the functional capacity of the bacterial community in the lower respiratory tract of rats. To further summarize the output of PICRUSt, we utilized BugBase, a bioinformatics tool that allows inference of community-wide phenotypes from predicted metagenomes ${ }^{32}$.BugBase found that gene function associated with anaerobiosis was enriched in the lower airway samples and, interestingly, gene function was reduced early in mechanical ventilation (CvsMV1 $P=0.013)$. The 
important genus attributed changed, with Proteobacteria being the dominant genus for 1hour of mechanical ventilation, and this dominance tended to decrease with time (Figure 6A). Biofilm formation and Gram-negative phenotypes were bacterial genus phenotypes frequently associated with respiratory pathogenicity. Our study suggested that biofilm formation phenotypes were substantially elevated during early tracheal intubation and mechanical ventilation, with Proteobacteria being the predominant cause of this phenotype. Moreover, Gram-negative phenotypes have no significant differences between groups, but phenotypes were more diverse in samples from 6hours of mechanical ventilation. Biofilm-forming bacteria were observed in the predicted metagenomes of each group. With the increasing understanding of the microbiota, we recognize interactions between the microbiota and the immune system of the airway epithelium. For example, asthma and allergy ${ }^{11}$ vary with the occurrence of airway infections. Studies have confirmed that microinhalation, external pressure, etc., favor the establishment of microbial biofilms. Biofilms have significant antimicrobial tolerance, evading host immune system attack and persisting in the host ${ }^{33}$. In the present study, biofilm-associated gene phenotypes were enhanced in rats undergoing mechanical ventilation with tracheal intubation, indicating enhanced biogenesis, consistent with previous studies ${ }^{34}$. Overall, these data suggest that the initial phase of mechanical ventilation by tracheal intubation disrupts the ecotone. Anaerobic phenotypic function decreased, and other phenotypes associated with bacterial pathogenicities also were influenced. However, it is also difficult to explain whether the reduced diversity of the microbiome is a causative factor for disease or whether diversity is simply a response to disease inflammation. 
bioRxiv preprint doi: https://doi.org/10.1101/2021.12.07.471701; this version posted December 9, 2021. The copyright holder for this preprint (which was not certified by peer review) is the author/funder. All rights reserved. No reuse allowed without permission.

259

260

261

262

263

264

265

266 
4. Discussion

268

269

With the continuous medical advances, tracheal intubation mechanical ventilation has been widely used in managing general anesthesia during surgery, intensive care units, and emergency resuscitation of critically ill patients. However, mechanical ventilation is also a "double-edged sword," which may lead to lung injury or aggravate existing lung injury ${ }^{35,36}$. Volume injury due to lung hyperinflation and shear injury due to repeated opening and closing of the terminal airway play an essential role in the pathogenesis of VILI ${ }^{3}$. On the other hand, respiratory inflammation may occur during ventilatory therapy. As an open cavity between the human body and the outside world, the respiratory tract has a normal microbiota that is an integral part of the human body's natural defense. Respiratory microbiota provides a biological barrier against the invasion of foreign bodies or pathogenic bacteria through mechanisms such as spatial occupancy, healthy competition, and secretion of antibacterial or bactericidal substances ${ }^{37-40}$. As one of the most frequently contacted parts of the human body, the respiratory micro-ecosystem is essential in maintaining human health and is also most vulnerable to damage. In recent years, the lower respiratory tract microbiota has been extensively studied and reported to be similar to that of the oropharynx $x^{1,2,41}$. However, studies exploring the relationship between mechanical ventilation and lower airway microecology have mainly explored at the level of ventilator-associated pneumonia (VAP) ${ }^{42-45}$. Changes in lower airway microecology during the initial phase of mechanical ventilation have not been reported. To investigate the in vivo response of tracheal intubation mechanical ventilation on lower respiratory microecology in SD rats in this study, we obtained pathological findings of lung inflammation and injury and data on bacterial community structure. 
In the present study, we observed that three hours after the administration of mechanical ventilation with tracheal intubation, hemorrhagic spots appeared in the lung tissue, widening of the alveolar septum, large amounts of exudate in the alveolar cavity, and altered alveolar structural damage, which aggravated with time. Correspondingly, the highest percentage of Proteobacteria was present in the experimental and control groups. At the onset of mechanical ventilation, the percentage of Proteobacteria in the lower airways increased more than twofold compared to controls. At the same time, the number of Firmicutes and Bacteroidetes decreased about fourfold. When six hours of mechanical ventilation had elapsed, Actinobacteria was more than four times that of the control group. These results suggest that mechanical ventilation with tracheal intubation alters the structure of the microecology while inducing lung tissue damage. However, how bacterial changes and tissue damage interact and require further study. We observed a significant amount of Acinetobacter within three hours of mechanical ventilation; therefore, Acinetobacter may be one of the factors involved in mediating the proinflammatory phase of lung tissue injury. It has been demonstrated that Acinetobacter may be the predominant bacteria in the lung microbiota of mechanically ventilated patients with pneumonia ${ }^{19}$. The excessive growth of this pathogen may promote airway injury and exacerbate microecological dysregulation. Lactobacillus and Streptococcus gradually increase with prolonged ventilation. Lactobacillus and Streptococcus act as probiotic strains. On the one hand, altering the composition of the host-microbiome dysregulate the microecology. On the other hand, they act indirectly through the standard mucosal immune system (CMIS) ${ }^{46}$. These probiotic strains may play a positive role in alleviating respiratory impairment and the corresponding microecological imbalance. In the mechanically ventilated 6-hour group, the main differential bacteria genus 
Prevotella_9 likewise belongs to probiotic bacteria and is often present in the intestine. Current studies

have confirmed the existence of an intestinal-pulmonary microbiome axis in the organism ${ }^{47}$. AS the metabolically active bacteria, Bacteroides and Firmicutes produce SCFA. We know that the gut microbiota can influence the microbiology of the lung through the distribution of SCFAs. SCFAs play a key role in maintaining mucosal immunity in the gut and lung tissues as a bridge between the microbiota and the immune system. ${ }^{22,23}$. Our findings may be relevant to understanding the mechanisms between tracheal intubation mechanical ventilation and lung tissue injury. Mechanical ventilation by tracheal intubation leads to an inflammatory response characterized by impaired airway defense mechanisms and alters in the microenvironment structure of the lower airways. In the future, we will further investigate probiotics to assess the prognostic relevance of microbial imbalance and the effectiveness of specific therapeutic interventions.

We analyzed lower airway microorganisms' alpha and beta diversity. We found that both tracheal intubation and mechanical ventilation cause changes in the microbial composition of the lower airways. At the beginning of tracheal intubation mechanical ventilation, bacterial species diversity decreased, indicating that intubation ventilation impaired the ecological niche balance in the lower airways of rats and disturbed bacterial diversity. Six hours after ventilation, bacterial diversity was elevated, and species composition remained different from healthy rats, considering that it may be related to pathogen overgrowth and stimulation of the host immune system that promotes disease development ${ }^{48}$. It is noteworthy that the experimental and control groups differed in bacterial species abundance but were more homogeneous. As seen in this study, the samples from the experimental and control groups were not well aggregated, suggesting that the overall community structure of the 
rat lower airway is influenced by tracheal intubation and mechanical ventilation. However, the sample

size of the groups in this experiment was small, and there were subject differences between individuals $^{37,39,49}$. The sample size needs to be increased in subsequent experiments to explore the relationship between the two further.

There may be a relationship between mechanical ventilation status and airway immune function.

Previous studies have shown that lower respiratory microbes are essential in the immune system's

maturation, education, and function. Especially, the regulation of the inflammatory response in respiratory diseases such as asthma ${ }^{50}$, chronic obstructive pulmonary disease (COPD) ${ }^{51}$, cystic fibrosis $^{52}$, and respiratory infections ${ }^{53}$ plays a key role. Ecological dysregulation leads to overgrowth of pathogenic bacteria, loss of symbiotic microbial diversity, and, ultimately, an inflammatory response in the host, leading to disease ${ }^{54} 55$. In the present study, after stimulation by mechanical ventilation with tracheal intubation, the microecological balance in the lower airways was disturbed, the loss of symbiotic microbial diversity, the immune system was affected, and inflammatory pathological changes began to develop in the lung tissue. The inflammatory cascade response and pro-inflammatory cytokines are hyperactivated with prolonged mechanical ventilation. Eventually, lung tissue increased damage. In BugBase functional analysis, we found biofilm formation gene-function phenotypes elevated abundance compared to the control group. It may be associated with damage to airway epithelial cells and disruption of defense mechanisms due to stimulation by tracheal intubation mechanical ventilation maneuvers. Biofilms have significant antimicrobial tolerance and can persist in the host and evade the host immune system ${ }^{33,56}$. We know that specialized respiratory epithelial cells are necessary to maintain immune homeostasis in the lower airway tract. As the first line of defense 
against potentially harmful environmental stimuli, respiratory epithelial cells are involved in maintaining immune homeostasis. Thus, epithelial cell dysfunction may influence the development of many airways and pulmonary inflammatory diseases ${ }^{57,58}$. In addition, it has been demonstrated that NF-kB, TINCR, and PGLYRP4 (rs3006458) are involved in airway immune mechanisms ${ }^{59,60}$. In future studies, we hope to investigate further the relationship between the mechanical ventilation status of tracheal intubation alone and epithelial cell alterations. We will make predictions of relevant metabolic pathways by metatranscriptomic metabolomic and proteomic studies.

In conclusion, our findings may be relevant to understanding the altered immune system mechanisms between tracheal intubation mechanical ventilation and lung tissue injury. Such operations as tracheal intubation ventilator-assisted ventilation lead to lung injury and inflammatory responses characterized by changes in the microbial community of the lower airways that become temporally correlated. The present study provides new ideas for functional studies on the role of pulmonary commensals in airway morphogenesis development, epithelial homeostasis, and the immune system. Particularly, it allows a more systematic study of bacteria's colonization and growth characteristics on pulmonary epithelial cells, their impact on lung infection and inflammation. Future studies hope to discover the effectiveness of new immunomodulatory or probiotic bacteria to prevent airway diseases associated with intraoperative or postoperative short-term intubation. 


\section{Reference :}

1. Huffnagle GB, Dickson RP, Lukacs NW. The respiratory tract microbiome and lung inflammation: a two-way street. Mucosal Immunol. 2017;10(2):299-306. doi:10.1038/mi.2016.108

2. Pragman AA, Lyu T, Baller JA, et al. The lung tissue microbiota of mild and moderate chronic obstructive pulmonary disease. Microbiome. 2018;6(1):7. doi:10.1186/s40168-017-0381-4

3. Brégeon F, Roch A, Delpierre S, et al. Conventional mechanical ventilation of healthy lungs induced pro-inflammatory cytokine gene transcription. Respir Physiol Neurobiol. 2002;132(2):191-203. doi:10.1016/s1569-9048(02)00069-1

4. Sigalet DL, Mackenzie SL, Hameed SM. Enteral nutrition and mucosal immunity: implications for feeding strategies in surgery and trauma. Can J Surg. 2004;47(2):109-116.

5. Charlson ES, Bittinger K, Haas AR, et al. Topographical continuity of bacterial populations in the healthy human respiratory tract. Am J Respir Crit Care Med. 2011;184(8):957-963. doi:10.1164/rccm.201104-0655OC

6. Segal LN, Alekseyenko AV, Clemente JC, et al. Enrichment of lung microbiome with supraglottic taxa is associated with increased pulmonary inflammation. Microbiome. 2013;1(1):19. doi:10.1186/2049-2618-1-19

7. Sze MA, Dimitriu PA, Hayashi S, et al. The lung tissue microbiome in chronic obstructive pulmonary disease. Am J Respir Crit Care Med. 2012;185(10):1073-1080. doi:10.1164/rccm.201111-20750C

8. Segal LN, Blaser MJ. A brave new world: the lung microbiota in an era of change. Ann Am Thorac Soc. 2014;11 
9. Dickson RP, Huffnagle GB. The Lung Microbiome: New Principles for Respiratory Bacteriology in Health and Disease. PLoS Pathog. 2015;11(7):e1004923. doi:10.1371/journal.ppat.1004923

10. Monsó E. Microbiome in chronic obstructive pulmonary disease. Ann Trans/ Med. 2017;5(12):251. doi:10.21037/atm.2017.04.20

11. Dickson RP, Erb-Downward JR, Huffnagle GB. The role of the bacterial microbiome in lung disease. Expert Rev Respir Med. 2013;7(3):245-257. doi:10.1586/ers.13.24

12. Dickson RP, Erb-Downward JR, Falkowski NR, Hunter EM, Ashley SL, Huffnagle GB. The Lung Microbiota of Healthy Mice Are Highly Variable, Cluster by Environment, and Reflect Variation in Baseline Lung Innate Immunity. Am J Respir Crit Care Med. 2018;198(4):497-508. doi:10.1164/rccm.201711-21800C

13. Cheng C, Li S, Wang Y, Chen S, You L, Zhang H. Ischemic postconditioning alleviates lung injury and maintains a better expression of aquaporin-1 during cardiopulmonary bypass. Chin Med $J$ (Engl). 2014;127(23):4012-4018.

14. dos Santos CC, Okutani D, Hu P, et al. Differential gene profiling in acute lung injury identifies injury-specific gene expression. Crit Care Med. 2008;36(3):855-865. doi:10.1097/CCM.0B013E3181659333

15. Guo M, Wu F, Hao G, et al. Bacillus subtilis Improves Immunity and Disease Resistance in Rabbits. Front Immunol. 2017;8:354. doi:10.3389/fimmu.2017.00354

16. Wickham H. ggplot2. WIREs Computational Statistics. 2011;3(2):180-185. doi:10.1002/wics.147 
17. Ondov BD, Bergman NH, Phillippy AM. Interactive metagenomic visualization in a Web browser. BMC Bioinformatics. 2011;12:385. doi:10.1186/1471-2105-12-385

18. Segata N, Izard J, Waldron L, et al. Metagenomic biomarker discovery and explanation. Genome Biol. 2011;12(6):R60. doi:10.1186/gb-2011-12-6-r60

19. Toma I, Siegel MO, Keiser J, et al. Single-molecule long-read $16 S$ sequencing to characterize the lung microbiome from mechanically ventilated patients with suspected pneumonia. $J$ Clin Microbiol. 2014;52(11):3913-3921. doi:10.1128/JCM.01678-14

20. Larsen JM, Steen-Jensen DB, Laursen JM, et al. Divergent pro-inflammatory profile of human dendritic cells in response to commensal and pathogenic bacteria associated with the airway microbiota. PLoS One. 2012;7(2):e31976. doi:10.1371/journal.pone.0031976

21. Larsen JM, Musavian HS, Butt TM, Ingvorsen C, Thysen AH, Brix S. Chronic obstructive pulmonary disease and asthma-associated Proteobacteria, but not commensal Prevotella spp., promote Toll-like receptor 2-independent lung inflammation and pathology. Immunology. 2015;144(2):333-342. doi:10.1111/imm.12376

22. Samuelson DR, Welsh DA, Shellito JE. Regulation of lung immunity and host defense by the intestinal microbiota. Front Microbiol. 2015;6:1085. doi:10.3389/fmicb.2015.01085

23. Dang AT, Marsland BJ. Microbes, metabolites, and the gut-lung axis. Mucosal Immunol. 2019;12(4):843-850. doi:10.1038/s41385-019-0160-6

24. Hill MO. Diversity and Evenness: A Unifying Notation and Its Consequences. Ecology. 1973;54(2):427-432. doi:10.2307/1934352 
25. Kembel SW, Cowan PD, Helmus MR, et al. Picante: R tools for integrating phylogenies and ecology. Bioinformatics. 2010;26(11):1463-1464. doi:10.1093/bioinformatics/btq166

26. Segal LN, Clemente JC, Tsay JCJ, et al. Enrichment of the lung microbiome with oral taxa is associated with lung inflammation of a Th17 phenotype. Nat Microbiol. 2016;1:16031. doi:10.1038/nmicrobiol.2016.31

27. Bauer TT, Torres A, Ferrer R, Heyer CM, Schultze-Werninghaus G, Rasche K. Biofilm formation in endotracheal tubes. Association between pneumonia and the persistence of pathogens. Monaldi Arch Chest Dis. 2002;57(1):84-87.

28. Gil-Perotin S, Ramirez $\mathrm{P}$, Marti $\mathrm{V}$, et al. Implications of endotracheal tube biofilm in ventilator-associated pneumonia response: a state of concept. Crit Care. 2012;16(3):R93. doi:10.1186/cc11357

29. Lozupone C, Knight R. UniFrac: a new phylogenetic method for comparing microbial communities. App/ Environ Microbiol. 2005;71(12):8228-8235. doi:10.1128/AEM.71.12.8228-8235.2005

30. El Kurdi R, Kumar K, Patra D. Introducing Principal Coordinate Analysis (PCoA) Assisted EEMF Spectroscopic Based Novel Analytical Approach for the Discrimination of Commercial Gasoline Fuels. J Fluoresc. 2020;30(6):1583-1589. doi:10.1007/s10895-020-02617-9

31. Langille MGI, Zaneveld J, Caporaso JG, et al. Predictive functional profiling of microbial communities using $16 \mathrm{~S}$ rRNA marker gene sequences. Nat Biotechnol. 2013;31(9):814-821. doi:10.1038/nbt.2676

32. Ward T, Larson J, Meulemans J, et al. BugBase Predicts Organism-Level Microbiome Phenotypes.; 2017. doi:10.1101/133462 
33. Lee AL, Goldstein RS. Gastroesophageal reflux disease in COPD: links and risks. Int J Chron Obstruct Pulmon Dis. 2015;10:1935-1949. doi:10.2147/COPD.S77562

34. Vandecandelaere I, Matthijs N, Van Nieuwerburgh F, et al. Assessment of microbial diversity in biofilms recovered from endotracheal tubes using culture dependent and independent approaches. PLoS One. 2012;7(6):e38401. doi:10.1371/journal.pone.0038401

35. Gajic O, Dara SI, Mendez JL, et al. Ventilator-associated lung injury in patients without acute lung injury at the onset of mechanical ventilation. Crit Care Med. 2004;32(9):1817-1824. doi:10.1097/01.ccm.0000133019.52531.30

36. Acute Respiratory Distress Syndrome Network, Brower RG, Matthay MA, et al. Ventilation with lower tidal volumes as compared with traditional tidal volumes for acute lung injury and the acute respiratory distress syndrome. N Eng/ J Med. 2000;342(18):1301-1308. doi:10.1056/NEJM200005043421801

37. Wu H, Kuzmenko A, Wan S, et al. Surfactant proteins A and D inhibit the growth of Gram-negative bacteria by increasing membrane permeability. J Clin Invest. 2003;111(10):1589-1602. doi:10.1172/JCI16889

38. Munyard P, Bush A. How much coughing is normal? Arch Dis Child. 1996;74(6):531-534. doi:10.1136/adc.74.6.531

39. Dickson RP, Martinez FJ, Huffnagle GB. The role of the microbiome in exacerbations of chronic lung diseases. Lancet. 2014;384(9944):691-702. doi:10.1016/S0140-6736(14)61136-3

40. Dickson RP, Erb-Downward JR, Huffnagle GB. Towards an ecology of the lung: new conceptual models of pulmonary microbiology and pneumonia pathogenesis. Lancet Respir Med. 2014;2(3):238-246. 
doi:10.1016/S2213-2600(14)70028-1

41. Bassis CM, Erb-Downward JR, Dickson RP, et al. Analysis of the upper respiratory tract microbiotas as the source of the lung and gastric microbiotas in healthy individuals. mBio. 2015;6(2):e00037. doi:10.1128/mBio.00037-15

42. Huang Y, Jiao Y, Zhang J, et al. Microbial Etiology and Prognostic Factors of Ventilator-associated Pneumonia: A Multicenter Retrospective Study in Shanghai. Clin Infect Dis. 2018;67(suppl_2):S146-S152. doi:10.1093/cid/ciy686

43. Luyt CE, Hékimian G, Koulenti D, Chastre J. Microbial cause of ICU-acquired pneumonia: hospital-acquired pneumonia versus ventilator-associated pneumonia. Curr Opin Crit Care. 2018;24(5):332-338. doi:10.1097/MCC.0000000000000526

44. Bailey KL, Kalil AC. Ventilator-Associated Pneumonia (VAP) with Multidrug-Resistant (MDR) Pathogens: Optimal Treatment? Curr Infect Dis Rep. 2015;17(8):494. doi:10.1007/s11908-015-0494-5

45. Di Pasquale M, Ferrer $M$, Esperatti $M$, et al. Assessment of severity of ICU-acquired pneumonia and association with etiology. Crit Care Med. 2014;42(2):303-312. doi:10.1097/CCM.0b013e3182a272a2

46. Thaiss CA, Zmora N, Levy M, Elinav E. The microbiome and innate immunity. Nature. 2016;535(7610):65-74. doi:10.1038/nature18847

47. Budden KF, Gellatly SL, Wood DLA, et al. Emerging pathogenic links between microbiota and the gut-lung axis. Nat Rev Microbiol. 2017;15(1):55-63. doi:10.1038/nrmicro.2016.142 
48. Fujimura KE, Lynch SV. Microbiota in allergy and asthma and the emerging relationship with the gut microbiome. Cell Host Microbe. 2015;17(5):592-602. doi:10.1016/j.chom.2015.04.007

49. Dreyfuss D, Saumon G. Ventilator-induced lung injury: lessons from experimental studies. Am J Respir Crit Care Med. 1998;157(1):294-323. doi:10.1164/ajrccm.157.1.9604014

50. Ling Z, Liu X, Cheng Y, Li L. Potential roles of disordered airway microbiota in patients with severe asthma. $J$ Allergy Clin Immunol. 2016;137(2):648. doi:10.1016/j.jaci.2015.09.043

51. Huang YJ, Erb-Downward JR, Dickson RP, Curtis JL, Huffnagle GB, Han MK. Understanding the role of the microbiome in chronic obstructive pulmonary disease: principles, challenges, and future directions. Trans/ Res. 2017;179:71-83. doi:10.1016/j.trsl.2016.06.007

52. Madan JC. Neonatal Gastrointestinal and Respiratory Microbiome in Cystic Fibrosis: Potential Interactions and Implications for Systemic Health. Clin Ther. 2016;38(4):740-746. doi:10.1016/j.clinthera.2016.02.008

53. Yin Y, Hountras P, Wunderink RG. The microbiome in mechanically ventilated patients. Curr Opin Infect Dis. 2017;30(2):208-213. doi:10.1097/QCO.0000000000000352

54. Haahtela T, Holgate S, Pawankar R, et al. The biodiversity hypothesis and allergic disease: world allergy organization position statement. World Allergy Organ J. 2013;6(1):3. doi:10.1186/1939-4551-6-3

55. Fujimura KE, Slusher NA, Cabana MD, Lynch SV. Role of the gut microbiota in defining human health. Expert Rev Anti Infect Ther. 2010;8(4):435-454. doi:10.1586/eri.10.14

56. Thurlow LR, Hanke ML, Fritz T, et al. Staphylococcus aureus biofilms prevent macrophage phagocytosis and 
attenuate inflammation in vivo. J Immunol. 2011;186(11):6585-6596. doi:10.4049/jimmunol.1002794

57. Hooper LV, Littman DR, Macpherson AJ. Interactions between the microbiota and the immune system. Science. 2012;336(6086):1268-1273. doi:10.1126/science.1223490

58. Hiemstra PS, McCray PB, Bals R. The innate immune function of airway epithelial cells in inflammatory lung disease. Eur Respir J. 2015;45(4):1150-1162. doi:10.1183/09031936.00141514

59. Igartua C, Davenport ER, Gilad Y, Nicolae DL, Pinto J, Ober C. Host genetic variation in mucosal immunity pathways influences the upper airway microbiome. Microbiome. 2017;5(1):16. doi:10.1186/s40168-016-0227-5

60. McAleer JP, Kolls JK. Contributions of the intestinal microbiome in lung immunity. Eur $J$ Immunol. 2018;48(1):39-49. doi:10.1002/eji.201646721 
Figure legend

Figure 1. Effect of mechanical ventilation on lung tissues injury from rats. (a) Rats were executed directly after tracheal intubation under anesthesia. (b-d) Rats were kept under anesthesia after tracheal intubation with spontaneous breathing for 1,3 , and 6 h. (e-g). Rats were mechanically ventilated for 1,3 , and $6 \mathrm{~h}$ after tracheal intubation. (h) Lung tissue injury score. Data are representative of at least four different experiments. Results are mean $\pm S$, for four rats. ${ }^{*} p<0.05$, ${ }^{* *} p<0.01,{ }^{* *} p<0.001$, compared with control animals or between mechanical ventilation six hours and spontaneous breathing six hours.

Figure 2. Changes in the lower respiratory tract flora of rats during mechanical ventilation. (a) At the phylum level, the main dominant phyla were Proteobacteria, Firmicutes, Bacteroidetes, Cyanobacteria, and Actinobacteria. (b) At the genus level, the main dominant genera were Acinetobacter, Lactobacillus, and Staphylococcus. Streptococcus. (c) LEFse analysis (LDA>2.0) was performed for all groups, with corresponding differential strains for control, MV6, SV6, Acinetobacter_radioresistens for control, Megamonas for MV6, and Parvibacter for SV6. In summary, tracheal intubation mechanical ventilation caused changes in the lower respiratory tract flora ratio in rats.

Figure 3. Alpha diversity of rat lower respiratory tract flora. (a) The observed species index decreased in the first 3 hours of mechanical ventilation and gradually increased as ventilation was prolonged. (b) Simpson index was not statistically different among groups, and the homogeneity of species was more consistent among groups. (c) Alpha diversity dilution curves leveled off in all groups, indicating a sufficient amount of data in each group. 
536 Figure 4. Beta diversity of rat lower respiratory tract flora. (a-c) In unweighted Unifrac analysis,

537 tracheal intubation mechanical ventilation made the rat's lower respiratory flora more fragmented. (d-f)

538 In the weighted Unifrac analysis of all groups, tracheal intubation mechanical ventilation did not make

539 the rat respiratory flora more dispersed. However, in comparing normal rats with the spontaneously

540 breathing group, a more significant dispersion of the rat lower respiratory flora occurred.

541 Figure 5. PICRUSt2 analysis of rat lower respiratory tract flora. Thirty-three unique KEGG pathways

among the inferred metagenomes. It is showed more remarkable similarities between normal rats and each ventilated group.

544 Figure 6. Bugbase analysis of rat lower respiratory tract flora. (a) With the associated representative 545 genera changes, anaerobic activity is diminished at the beginning of mechanical ventilation by tracheal

546 intubation. (b) Forms_Biofilm is enhanced at the beginning of mechanical ventilation by tracheal

547 intubation, and the associated representative genera are altered. (c) Gram_Negative formation did not vary much between groups, slightly increased at the beginning of the experiment, and the proportion 549 slightly decreased with the prolongation of the experiment. 
553 Table 1. Effect of mechanical ventilation on lung wet to dry weight ratio (W/D ) from rats $(n=4, \bar{X} \pm S D)$ 554

\begin{tabular}{llllllll}
\hline & \multirow{2}{*}{ Control } & \multicolumn{3}{c}{ Mechanical Ventilation } & \multicolumn{3}{c}{ Spontaneously Breathing } \\
\cline { 3 - 8 } & & $1 \mathrm{~h}$ & $3 \mathrm{~h}$ & $6 \mathrm{~h}$ & $1 \mathrm{~h}$ & $3 \mathrm{~h}$ & $6 \mathrm{~h}$ \\
\hline W/ & $5.579 \pm 0.39$ & $5.958 \pm 0.56$ & $6.839 \pm 1.04$ & $11.618 \pm 1.63^{*}$ & $5.873 \pm 0.28$ & $6.895 \pm 0.16$ & $8.561 \pm 1.34$ \\
D & 3 & $9^{\#}$ & $7^{\#}$ & $\#$ & $1^{\#}$ & $3^{\#}$ & $8^{*}$ \\
\hline
\end{tabular}

556 Data are means and(sd) for control, Mechanical Ventilation(1, 3, $6 \mathrm{~h})$ and Spontaneously Breathing (1, 5573,6 h) rats.

558 Value are mean \pm SD.

$559{ }^{*} \mathrm{p}<0.05$ vs control. $\# p<0.05$ vs mechanical ventilaition 6 


\section{Funding}

562 This study was supported by the Guangdong Provincial Science and Technology Project (NO.

563 2017B020235001)

\section{Acknowledgements}

566 We thank the Animal Experimentation of Nanfang Hospital, Southern Medical University (Guangzhou, 567 China) for performing animals and experimental sites. We are grateful for the sequencing platform or 568 bio information analysis of Gene Denovo Biotechnology Co., Ltd (Guangzhou, China). 
1 Figure

2 Figure 1.
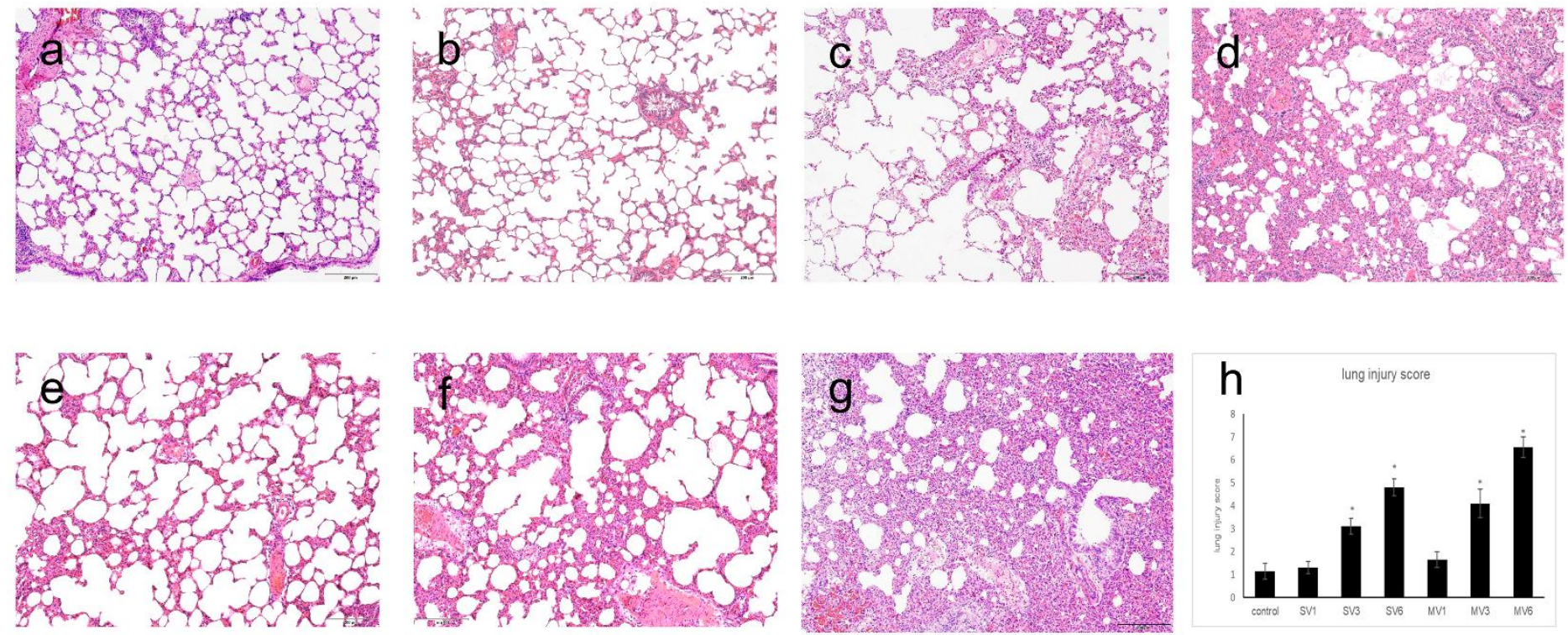

4 Figure 1. Effect of mechanical ventilation on lung tissues injury from rats. (a) Rats were executed directly after tracheal intubation under anesthesia. (b-d) Rats were kept under anesthesia after tracheal intubation with spontaneous breathing for 1, 3, and $6 \mathrm{~h}$. (e-g). Rats were mechanically ventilated for 1, 3, and $6 \mathrm{~h}$ after tracheal intubation. (h) Lung tissue injury score. Data are representative of at least four different experiments. Results are mean $\pm S$, for four rats. ${ }^{*} p<0.05$, ${ }^{* *} p<0.01,{ }^{* *} p<0.001$, compared with control animals or between mechanical ventilation six hours and spontaneous breathing six hours. 
a

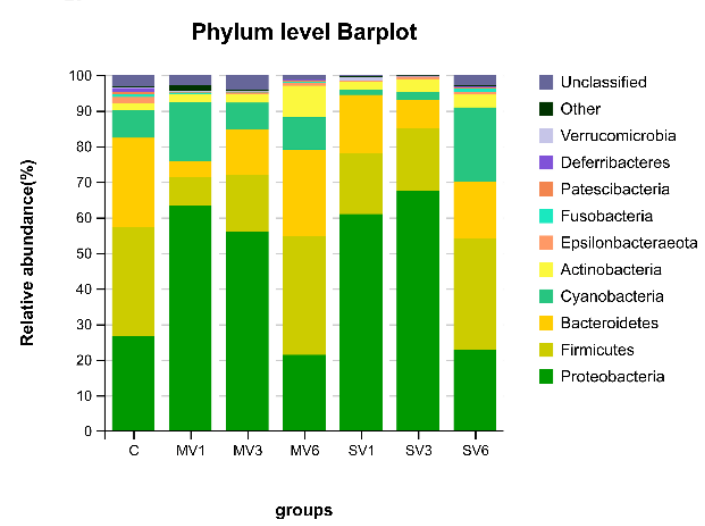

b

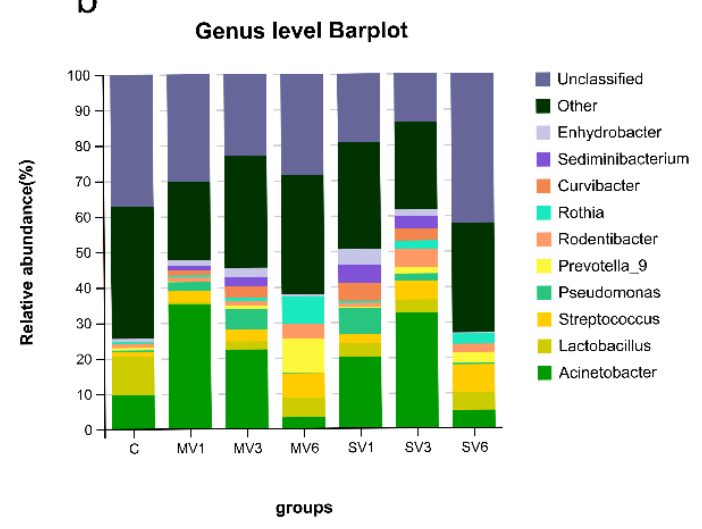

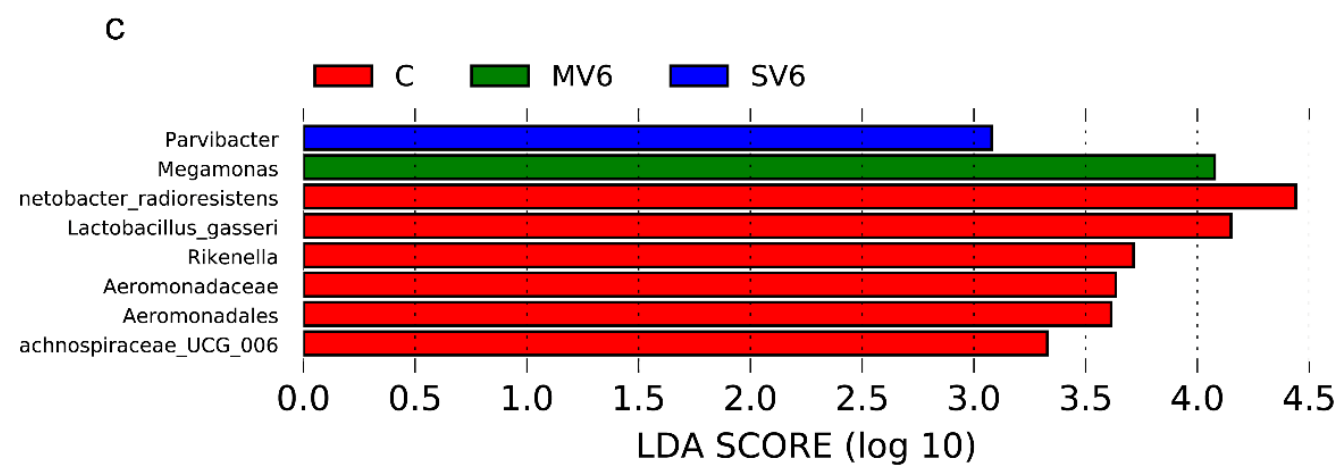

Figure 2. Changes in the lower respiratory tract flora of rats during mechanical ventilation. (a) At the phylum level, the main dominant phyla were Proteobacteria, Firmicutes, Bacteroidetes,

17 Cyanobacteria, and Actinobacteria. (b) At the genus level, the main dominant genera were

Acinetobacter, Lactobacillus, and Staphylococcus. Streptococcus. (c) LEFse analysis (LDA>2.0) was performed for all groups, with corresponding differential strains for control, MV6, SV6,

Acinetobacter_radioresistens for control, Megamonas for MV6, and Parvibacter for SV6. In summary,

21 tracheal intubation mechanical ventilation caused changes in the lower respiratory tract flora ratio in rats. 
Figure3

a

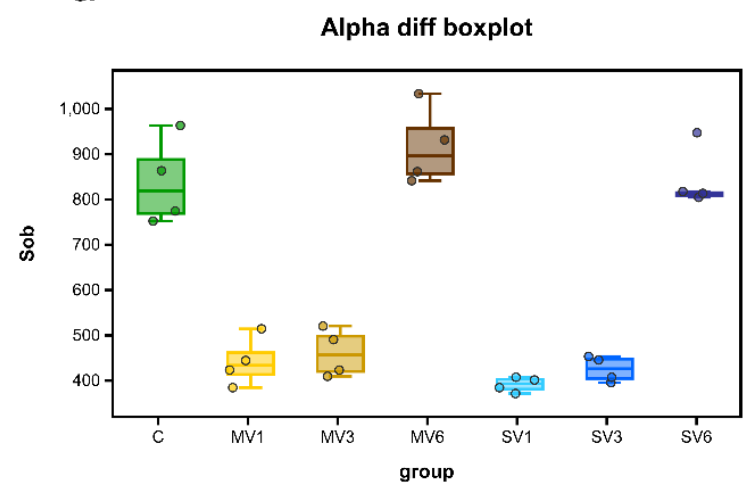

b

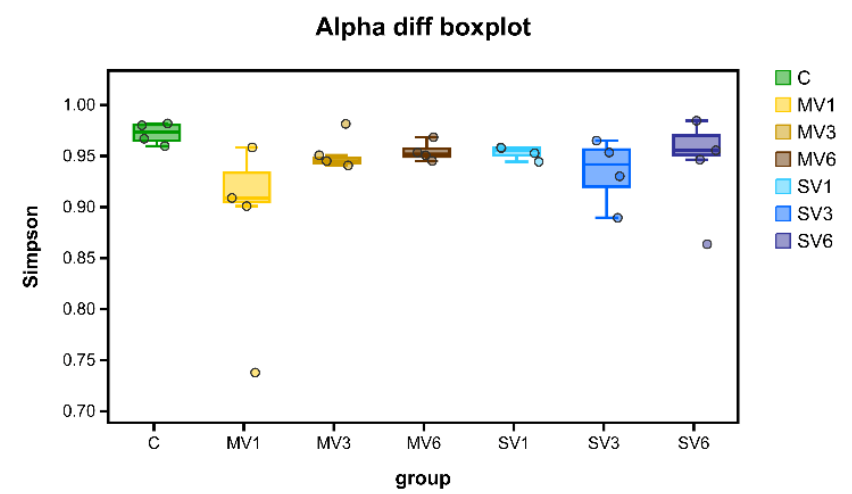

C

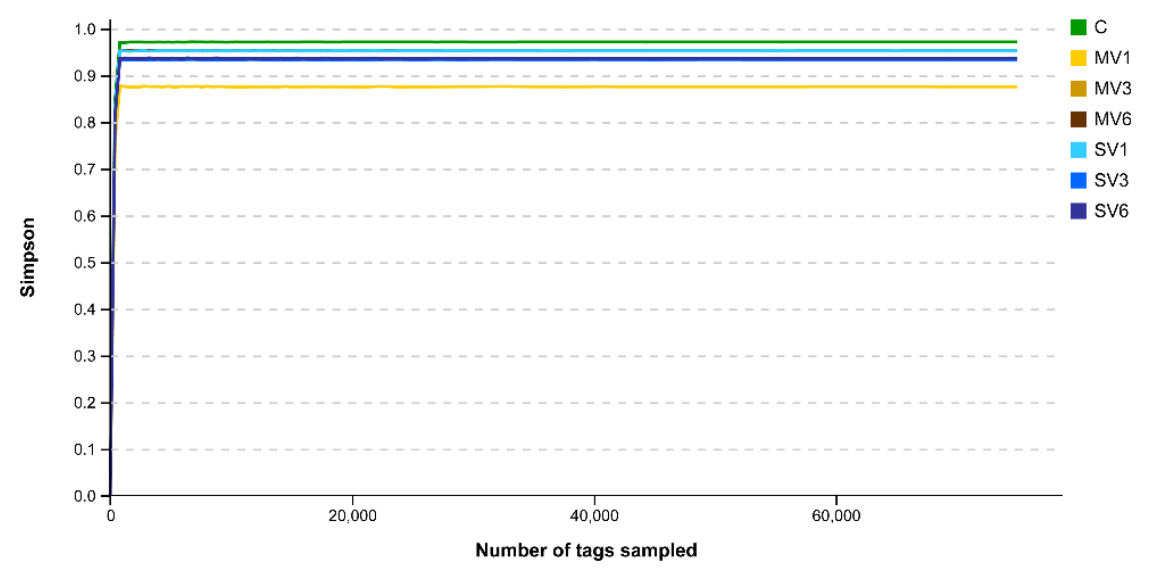

25 Figure 3. Alpha diversity of rat lower respiratory tract flora. (a) The observed species index

26 decreased in the first 3 hours of mechanical ventilation and gradually increased as ventilation was

27 prolonged. (b) Simpson index was not statistically different among groups, and the homogeneity of

28 species was more consistent among groups. (c) Alpha diversity dilution curves leveled off in all

29 groups, indicating a sufficient amount of data in each group. 
Figure 4
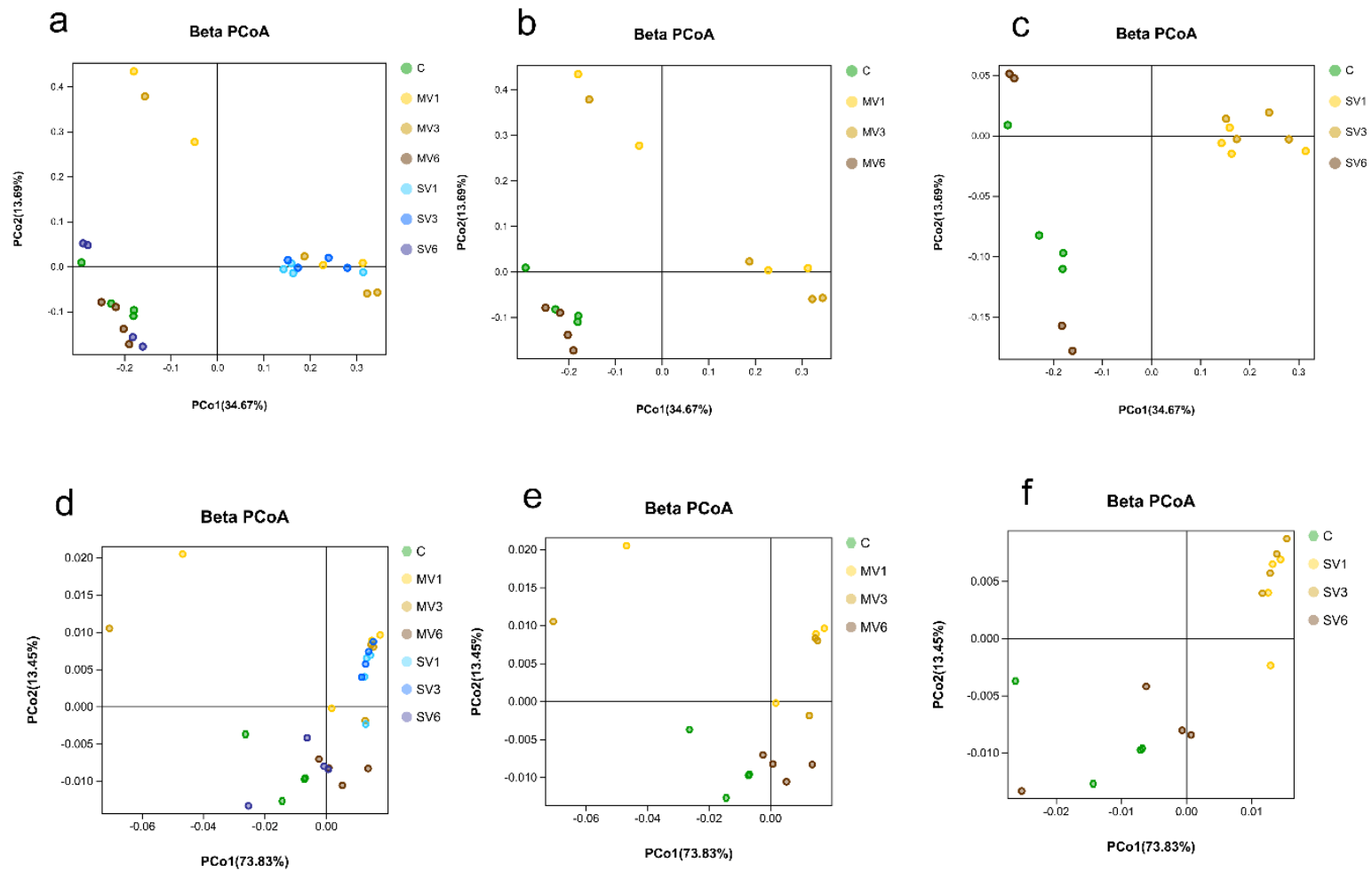

Figure 4. Beta diversity of rat lower respiratory tract flora. (a-c) In unweighted Unifrac analysis, tracheal intubation mechanical ventilation made the rat's lower respiratory flora more fragmented. (d-

f) In the weighted Unifrac analysis of all groups, tracheal intubation mechanical ventilation did not make the rat respiratory flora more dispersed. However, in comparing normal rats with the spontaneously breathing group, a more significant dispersion of the rat lower respiratory flora occurred. 
Figure 5.

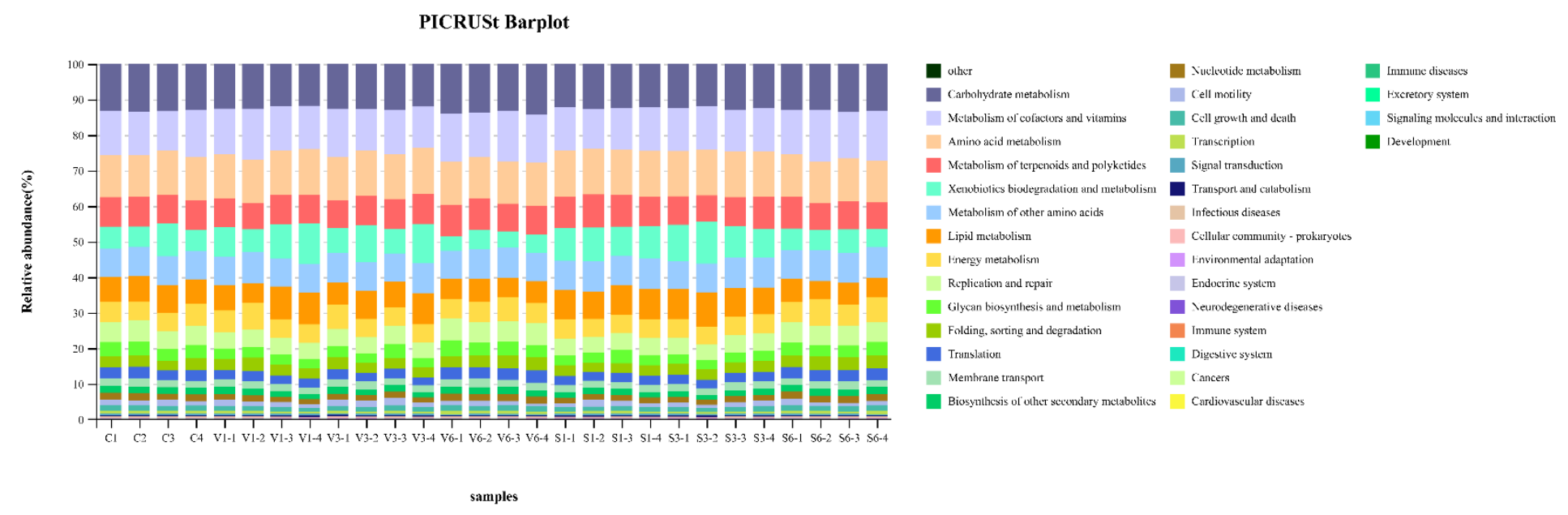

Figure 5. PICRUSt2 analysis of rat lower respiratory tract flora. Thirty-three unique KEGG pathways 
Figure 6.
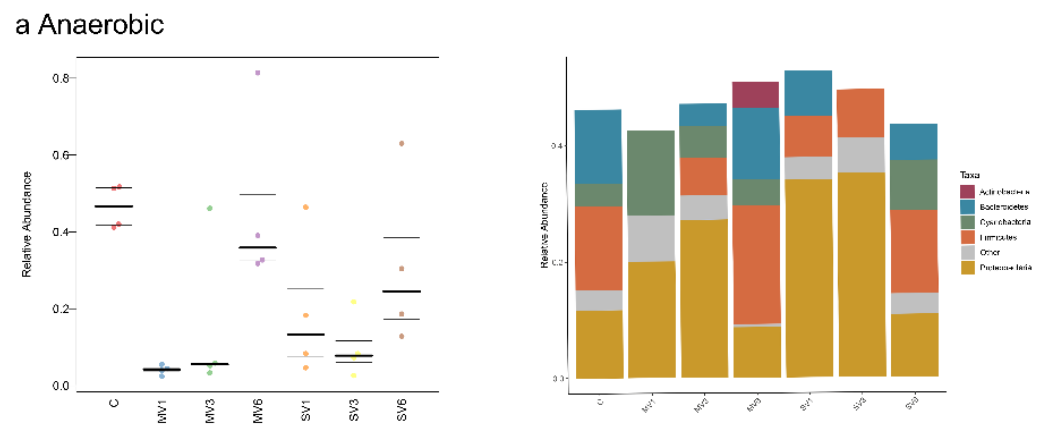

b Forms_Biofilms
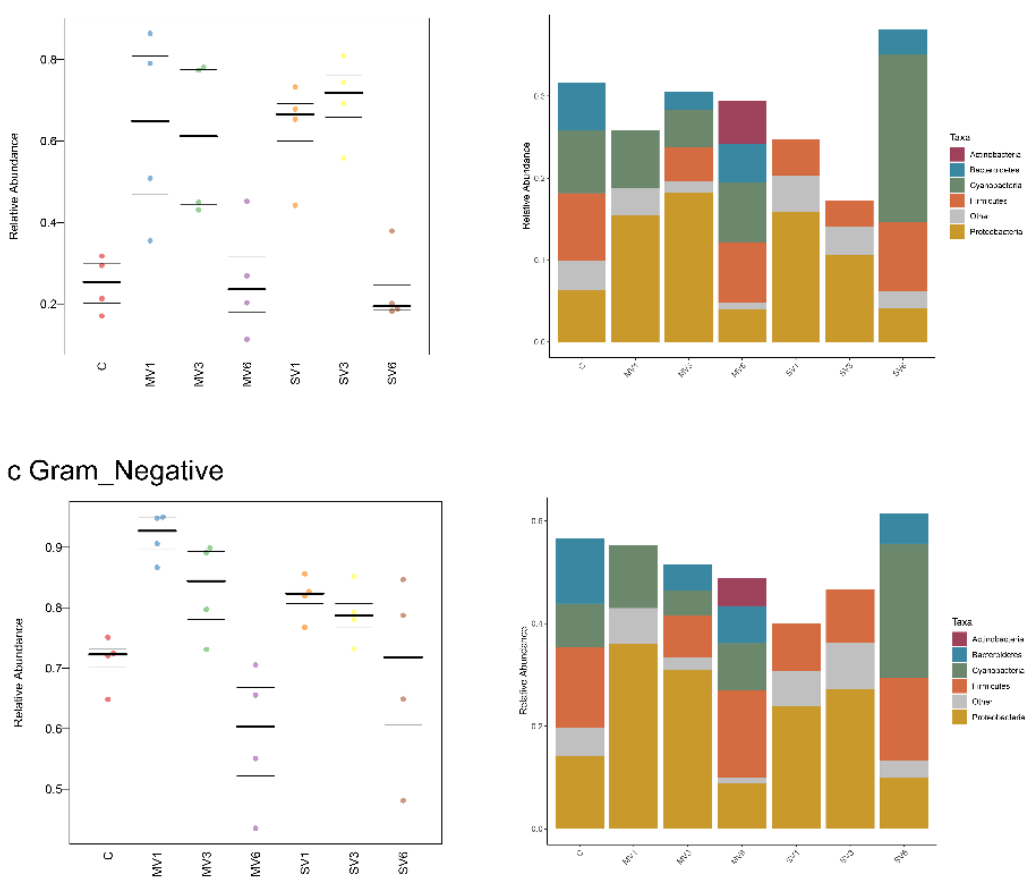

60 Figure 6. Bugbase analysis of rat lower respiratory tract flora. (a) With the associated representative 61 genera changes, anaerobic activity is diminished at the beginning of mechanical ventilation by 62 tracheal intubation. (b) Forms_Biofilm is enhanced at the beginning of mechanical ventilation by 63 tracheal intubation, and the associated representative genera are altered. (c) Gram_Negative 64 formation did not vary much between groups, slightly increased at the beginning of the experiment, 65 and the proportion slightly decreased with the prolongation of the experiment. 